\title{
Different Explanations for the Cosmic Microwave Background Radiation
}

\author{
Ting-Hang Pei \\ Institute of Astronomy and Astrophysics, Academia Sinica, Taiwan \\ thpei@asiaa.sinica.edu.tw; Thpei142857@gmail.com
}

\begin{abstract}
In this research, the other reasonable explanations for the cosmic microwave background radiation is revealed. Due to the microwave resolution, it very roughly shows the image of galaxies in the universe. Moreover, the intensity measurement on each pixel of the image is the sum of the incident microwaves from different directions, so the microwave image cannot represent the microwave sources clearly far away from the Earth. Hence, we propose a simulation after removing several strongest microwave sources, the remaining microwave radiation sources can establish a very uniform intensity distribution over a range of several ten light years. On the other hand, Sloan Digital Sky Survey reveals 200 million galaxies in the universe and, in fact, only to eliminate the contributions of all galaxies from the microwave image is impossible. The way to further obtain the fine-scale structure by only removing the few strongest microwave sources as the foreground effect will keep the other contributions from all the rest galaxies and stars. Therefore, the Cosmic Microwave Background cannot be uniquely explained the radiation which was left after the initial formation of the universe. Moreover, it is the mainly residual radiation from the un-calculated galaxies and inaccurate estimation of the microwave source strength.
\end{abstract}

Keywords: Cosmic Microwave Background, Cosmic Infrared Background, Cosmic Background Detector, Planck Surveyor, Sloan Digital Sky Survey, Baryon Oscillation Spectroscopic Survey

CMB - Cosmic Microwave Background

COBE - Cosmic Background Detector

WMAP - Wilkinson Microwave Anisotropy Detector

ESA - European Space Agency

CIB - Cosmic Infrared Background

FIRB - Far-Infrared Background

BICEP - Background Imaging of Cosmic Extragalactic Polarization

SDSS - Sloan Digital Sky Survey

BOSS - Baryon Oscillation Spectroscopic Survey

\section{Introduction}

The CMB [1-3] is regarded as the radiation left by the early development of the universe. It is thought as the microwave electromagnetic radiation filling the entire universe after the "big bang" in cosmology. CMB is nearly identical in all directions, but subtle residual changes exhibit tiny anisotropy [2]. In the past fifty years, people made a lot of measurements and detailed research on CMB in different bands, and found that it meets well with the blackbody radiation spectrum in a fairly wide range of wavelengths, corresponding to a temperature of $2.725 \mathrm{~K}$ [3].

A well-known experiment was proceeded by the National Aeronautics and Space Administration's COBE satellite, which detected large-scale microwave image with 
limited detection capabilities [4]. However, COBE did not have good resolution to perform anisotropic experiments on small angular scales. Then the sky-viewing experiments using balloons near the ground were investigated more delicately to an angular scale of about 1 degree [4]. At the same period, WMAP measured the largescale anisotropy of the entire sky more accurately and gave detailed measurements of the angular power spectra at a scale less than 1 degree [4,5]. In the following, the European Space Agency's Planck satellite proceeds a more detailed investigation until now [4]. However, the original $\mathrm{CMB}$ data collected from satellites was thought to contain foreground effects that completely cover the fine-scale structure of the cosmic microwave background. Scientists considered that the Milky Way and extragalaxies provide microwave sources which must be subtracted to reveal this fine-scale structure, the so-called cosmic anisotropic microwave background. However, the detailed analysis is complicated and difficult to calculate. In practice, it is very difficult to account all the microwave sources in the universe.

Extracting cosmology science must carry out signal-foreground separation detailed where the six main foreground models motivated by Planck data are summarized [6]. Two CIB spectra have been revealed [6,7]. Recently, various aspects of the CMB spectral distortions have been reviewed [8], and different factors causing the distortions are also briefly introduced, such as: Bose-Einstein ( $\mu$-type) distortions, weak Comptonization (y-type) distortions, intermediate epoch distortions, and sources of spectral distortions. These distortion factors gradually become widespread in discussions. In addition, the latest evidence of intergalactic medium heating from active galactic nuclei feedback has also been studied [8]. Not only the aforementioned distortion sources, but the plasma affecting the $\mathrm{CMB}$ radiation in a variety of quantifiable ways has also been discussed [9]. Furthermore, the Planck CMB anisotropic power spectra show that the primordial black holes with mass $m_{\mathrm{BH}} \gtrsim 5 \mathrm{M} \odot$ are disfavored for constraints [10]. However, this is susceptible to sizable uncertainties in treating the black-hole accretion process [10]. Besides, the hot gas in unresolved groups of galaxies is also predicted to lead the spectral distortions [11-13]. The distortion of the CMB Spectrum from the intergalactic dust has also been reported to an upper limit of $0.06 \%$ on the FIRB contributions [14]. When more and more possible sources of distortions are discovered, the spectrum of CMB is more and more different from the earlier results. This also shows that some things have not been discovered which may have potential to affect the CMB spectrum. These messages also support our discussion in this paper.

Another related example can illustrate the results of incorrect estimates of radiation sources. In 2014, the BICEP2 team claimed to detect a large-scale B-mode polarization signal from the Big Bang [15], but the estimation was wrong because the intensity of 
the unknown foreground noise [16]. The weak gravity lensing signal would have a stronger E-mode signal where the signal mixes with the B-mode signal [16]. Later, in collaboration with the Planck team $[17,18]$, it was concluded that most of the measured B-modes came from the polarized dust of the Milky Way. The follow-up results of Planck satellites and the joint analysis of BICEP2/Keck and Planck data show that this interpretation is incorrect, and galactic dust accounts for most of the BICEP2 signal, while the amount of gravitational waves from inflation can only be set an upper limit [16]. It is even clearly stated that a signal in the cosmic microwave background can be explained by interstellar dust when this signal is thought to be evidence of inflation in the early universe [19].

However, as we know, the microwave source is a long-wavelength source in $\mathrm{cm}$ range. Using it to do imaging reconstruction naturally exists the substantial resolution problem [20,21]. Even the higher frequency in the Terahertz region, the imaging resolution is still a problem [22-25]. How to improve the resolution has been proceeded for many years. According to the cosmic map produced by SDSS including new data from BOSS [26,27], at least 200 million galaxies [26-29] exist in the universe, and the microwave contributions of the most galaxies show on each pixel of the cosmic microwave image. In fact, the microwave imaging is not as clear as the X-Ray imaging. The wavelength of the microwave is in the $\mathrm{cm}$ range, and the image edges are blurred for the detected body [3,20-24]. Traditionally, the microwave detector is the horn antenna and the receiving port is an opening with a finite area [30-34]. Due to the resolution limitation of the microwave imaging, it causes our curiosity whether the traditional CMB explanation is reasonable or not? Why is there a uniform microwave distribution in the sky? In the 1987A supernova event [35], the generated light travels in the universe at the speed of light and reach the Earth after 168,000 years. Similar to this, it makes us doubt whether it is still possible to measure the microwave radiation of 13.8 billion years ago since the Big Bang? Just as it takes eight minutes and eighteen seconds for sunlight traveling to the Earth, electromagnetic waves or photons pass through space between galaxies and galaxies at the speed of light. Once they are generated, they start to move forward to the edge of the universe. if no absorption by other substances, the microwaves generated 13.8 billion years ago have already traveled a distance of 13.8 billion light-years. Therefore, how can the empty universe reserve and keep the microwave radiations almost moving straightly at the speed of light since 13.8 billion years ago? Based on those reviews, we use several viewpoints to discuss this topic in this research. A picture taken on 2009 Tokyo Christmas Festival [36] also helps us to understand some concepts here. 


\section{The New Explanation of The Cosmic Microwave Imaging}

As we know, as long as the temperature is higher than the absolute zero, there are black-body radiation and the corresponding spectrum. The cosmic background radiation is obtained by using the microwave measurement to draw an omnidirectional image, and it shows the final result of the radiation homogenization at $2.7 \mathrm{~K}$ in all directions after complicated elimination of the foreground contamination. However, before obtaining the final results, we have to ask how much the information are clearly from the microwave imaging and what does it mean from those images?

If we measure the blackbody radiation spectrum of the sun, then it corresponds to about $6000 \mathrm{~K}$ of blackbody radiation. The use of satellites to measure the blackbody radiation in outer space may directly detect the blackbody radiation spectrum of the extremely thin cold gas in the universe, so the blackbody radiation measurement of the COBE satellite is consistent with the theoretical prediction almost without errors.

The Wilkinson Microwave Anisotropy Probe (WMAP) was responsible for the detection of the anisotropy in cosmic microwave radiation [5]. Two five-year images at $23 \mathrm{GHz}$ and $94 \mathrm{GHz}$ are shown in Fig. 1. It shows a lot of red regions at $23 \mathrm{GHz}$ in Fig. 1(a) and few red regions at $94 \mathrm{GHz}$ in Fig. 1(b). Comparing Fig. 1(b) with 1(a), it clearly reveals more detail about the universe what we understand. This big difference is due to the resolution that the detecting wavelength in Fig. 1(b) is shorter than Fig. 1(a). This full-sky map of the CMB contains 3,145,728 pixels [5] and each pixel includes the contribution of many illuminators. According to the largest 3D cosmic map [29], at least 200 million galaxies exist in the universe and each galaxy averagely contains about 100 200 billion stars. Some part of the 3D map is shown in Fig. 2(a) and each bright point in the dense map represents one galaxy in Fig. 2(b). It means an average of 660 contributory galaxies in each pixel so, roughly speaking, total about $6.6 \times 10^{14}$ to $1.32 \times 10^{15}$ stars averagely containing in each pixel. In fact, each pixel includes a lot of illuminators from different directions more than above values and their contributions are summarized to show on the microwave images. Even using $94 \mathrm{GHz}$ microwave to reconstruct the full-sky imaging, the resolution is still far less than the truth. Actually, the microwave images in Fig. 1 are very rough ones. Here we face one problem is the convincing information extracting from these images. Especially, CMB claims our universe is flat but it directly contradicts the truth as shown in Fig. 2.

According to above initial analysis, two more reasonable explanations are proposed in this research. The first explanation extends the original model that the radiation spectrum comes from the generations 13.8 billion years ago. If so, all the galaxies even billions of light years far away from the Earth have similar effects and their contributions have to be considered in the microwave images as mentioned previously. Especially, each galaxy has powerful contributions in the microwave range, from K- 
band to W-band. It has been proven in Fig. 1(b) that the strong microwave contributions from our galaxy, and the $23 \mathrm{GHz}$ image in Fig. 1(a) reveals the main microwave source is still from our galaxy but much wider than it is in $94 \mathrm{GHz}$ image. The measured spectrum is the superposition of all illuminators, and each microwave image shows the contributions from all galaxies, more than 200 billion galaxies revealing by SDSS. However, due to the resolution, it is not possible to make very detailed images like Xray imaging. The wavelength of the microwave is in the $\mathrm{cm}$ range, and the image edges are blurred for the detected body [3,20-24]. Therefore, it is impossible to accurately distinguish the galaxy shape several billion light-years away as good as the Hubble telescope. The full-sky microwave detection is equivalent to reconstruct images of many $\mu \mathrm{m}$-sized bodies using a source of wavelength in $\mathrm{cm}$, and the value at each point is a relatively large-scale average. However, CMB was obtained by using the optimal fitting to eliminate the foreground radiation, where only a few important microwave sources are considered. Here we immediately meet one question is how to correctly describe the foreground radiation from 200 million galaxies? Actually, it is impossible for us to give exact foreground radiation as a function of all galaxies. Microwaves coming from the far-distance galaxies is like from a light point source. When the foreground radiation is eliminated by using only few main microwave sources, the contributions of other galaxies are still left no matter how weak it is. Finally, it gives almost the same value at each imaging pixel by using the microwave detection. If CMB really exists, then those contributions from all galaxies are also recorded in the CMB image.

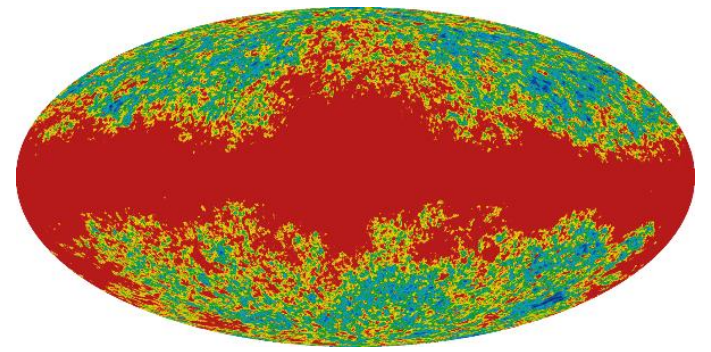

(a)

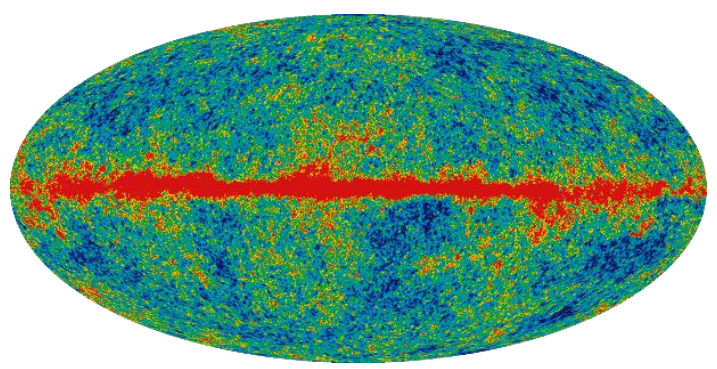

(b)

Figure 1. The five-year temperature maps by WMAP at observing frequencies (a) $23 \mathrm{GHz}$ and (b) 94 $\mathrm{GHz}[5]$.

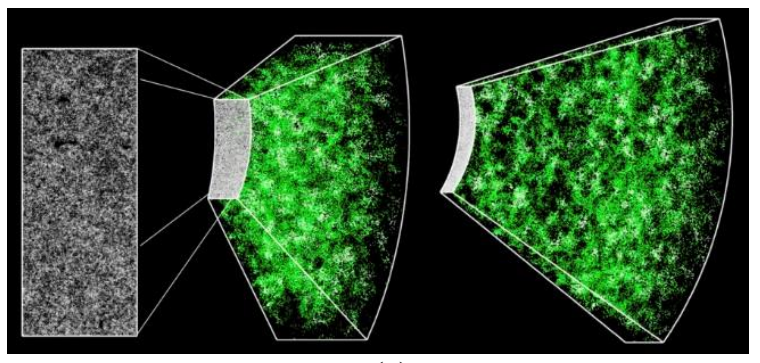

(a)

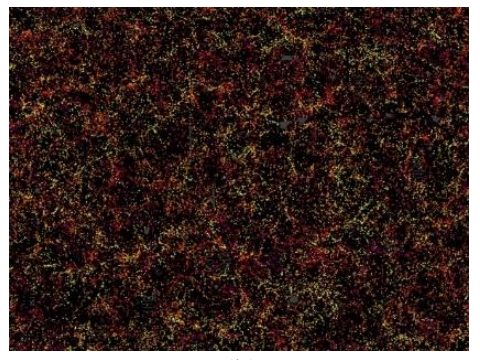

(b)

Figure 2. (a) The part of 3D universe map from the Sloan Digital Sky Survey III, and 10 percent of Sloan Digital Sky Survey dataset show here [29]. (b) The dense map shows some parts of galaxies in our universe. Each bright point represents one galaxy [29]. 
In the following, a simulation is used to verify our viewpoint. We consider a cubic space of 5 million light years in each side and a square detecting plane of 500 thousand light years in each side. This square plane is parallel to one surface of the cubic space, and the distance between the square detecting plane and the nearest surface of the cubic space is 5 thousand light years. Both the centers of the cubic and the square detecting plane are on the same line parallel to the normal direction of the square detecting plane. Now, supposing $10^{4}$ galaxies randomly distribute in this cubic space and their radiated microwave intensities are also random. A random distribution of galaxies is shown in Fig. 3(a). If the space is reduced as shown in the picture, you can see that the distribution density is very high. When enlarged into a cube space of 5 million light-years, it will be very empty when viewed with the naked eye on the Earth. Each galaxy is treated as a point source in simulations and the intensity measured in space point is inversely proportional to the square distance from the point source to the space point. Then the microwave intensity at certain frequency $v$ in the square detecting plane is the contributions of all point sources, that is,

$$
\mathrm{I}(x, y)=\sum_{i} \frac{A_{i}\left(x_{i}, y_{i}, z_{i}\right)}{\left[\left(x_{i}-x\right)^{2}+\left(y_{i}-y\right)^{2}+z_{i}^{2}\right]},
$$

where $\left(x_{i}, y_{i}, z_{i}\right)$ is the coordinates of the point source and $(x, y, z=0)$ is the coordinate of the point on the square detecting plane. The intensity of each point source is described by $A_{i}$ in arbitrary unit, so we can choose $A_{i}$ is a random number between 0 and 1 in simulations for convenience. In particular, 10 other point light sources with intensities between 1 and $10^{6}$ are randomly selected as the extremely strong point light sources like the cosmic microwave image in Figure 1, in which some light sources are particularly strong. The coordinates of the 10 point light sources are also arbitrarily selected in this limited space, and the microwave intensity is calculated together with the contributions of the $10^{4}$ point light source. In the square detecting plane, the intensity distribution is not homogeneous and the maximum is about 3 times larger than the minimum as shown in Fig. 3(b). When the observer places in the vicinity of the maximum in Fig. 3(b), he will see the strong light intensity from all galaxies. On the contrary, he will see low light intensity when he is in the vicinity of the minimum in Fig. 3(b). If these strongest ten sources are removed from the intensity contribution, it will not become zero because of the non-zero contribution from the rest $10^{4}$ galaxies. The rest intensity distribution shown in Figure 3(c), where the difference between the maximum and minimum values distributed in a square area of 500,000 light years is only $10 \%$. It is a very good approximation to say the homogeneous microwave background established by the rest galaxies. However, the maximum decreases sharply from about 6,600 in Fig. 3(b) to only about 2.2 in Fig. 3(c). The drop was as much as 
3,000 times. Just like in the process of extracting CMB, after removing the main foreground pollutions, we get a fairly uniform microwave background value. It is just that the source of this very approximately uniform microwave background here is established by $10^{4}$ galaxies, which is not established from an event after a big supernova similar to the big bang in the beginning of the universe. The so-called CMB radiation now is due to the rest galaxies causing this result. When our solar system is placed on this square detecting plane, it only occupies very small area less than one-pixel size in Figs. 3(a) and (b). Comparing Fig. 3(b) with Fig. 3(c), because these 10 extremely strong light sources are added, the lowest value of the intensity distribution on the detection plane can be increased by several thousand times. Therefore, the method of removing the strongest light source will leave the uniform contribution of the remaining small galaxies. The intensity distributions of the other three groups of different small galaxies and 10 small galaxies of strong light sources are shown in Figs. 3(d), (f), and (h). In Fig. 3(d) the ratio of the maximum to minimum is about 1.4, the ratio is about 1.7 in Fig. 3(f), and the ratio is about $3.0 \mathrm{in} \mathrm{Fig.} \mathrm{3(h).} \mathrm{After} \mathrm{subtracting} \mathrm{the} 10$ strongest light sources, the difference between the maximum and minimum is about $8 \%$ in Fig. $3(\mathrm{e})$, the difference is about $6 \%$ in Fig. 3(g), and the difference is about $14 \%$ in Fig. 3(i). This means that different $10^{4}$ galaxies can produce different intensity fluctuations. But in many simulations, the average intensity in any unit falls around 2.0 overall, which can be seen from Figs. 3(c), (e), (g), and (i). That is to say, our simulations are statistically meaningful, and all of them can get close to a uniform background value. Moreover, our results are also scalable including the $A_{i}$, which means that the spatial distance and the size of the detection plane are reduced by a certain value at the same time. For example, when the reduction value is 5000, the two locally maximum peaks in Fig. 3(g) may correspond to the observed part of the anisotropy in CMB. In other words, the limited spatial distribution of our $10^{4}$ galaxies can not only explain the uniform $\mathrm{CMB}$ value in all directions, but also show the conclusion that anisotropy may exist. Here, we give another source to explain the observed phenomenon of CMB. Therefore, there are more than 20 billion galaxies in the universe, and their contribution in the microwave frequency band can explain the observation results of CMB from the Earth or orbiting satellites, and it can also explain the appearance of local inhomogeneous. 


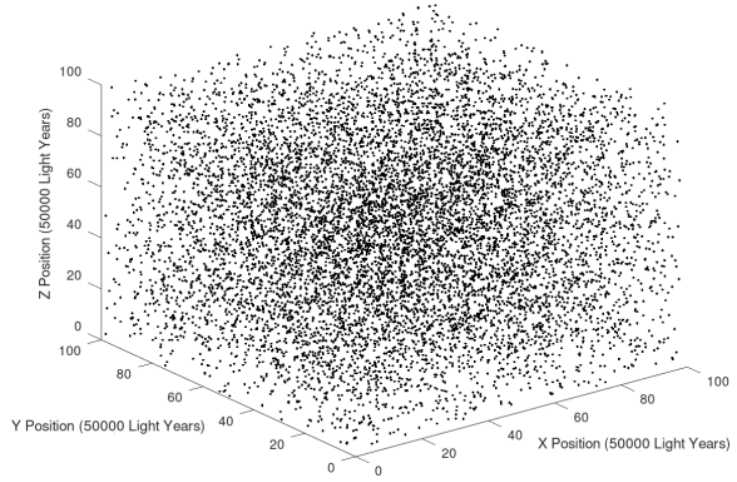

(a)

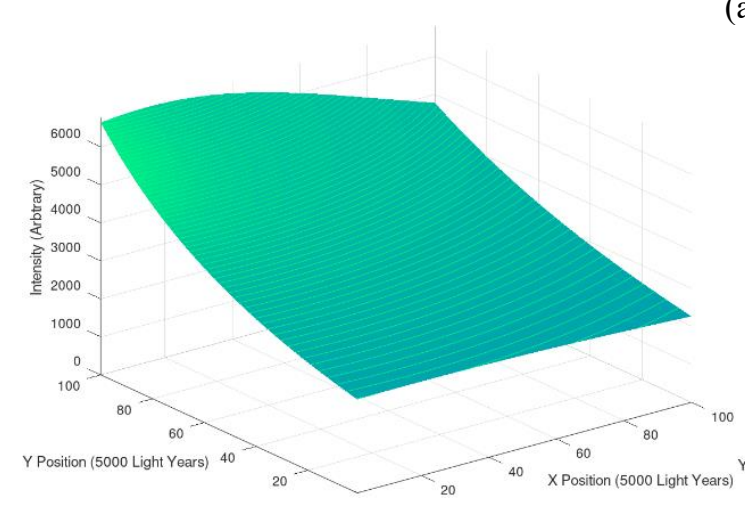

(a)

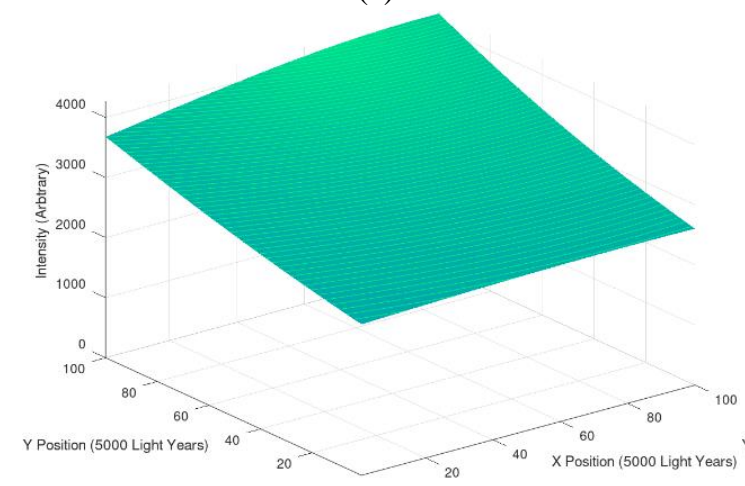

(d)

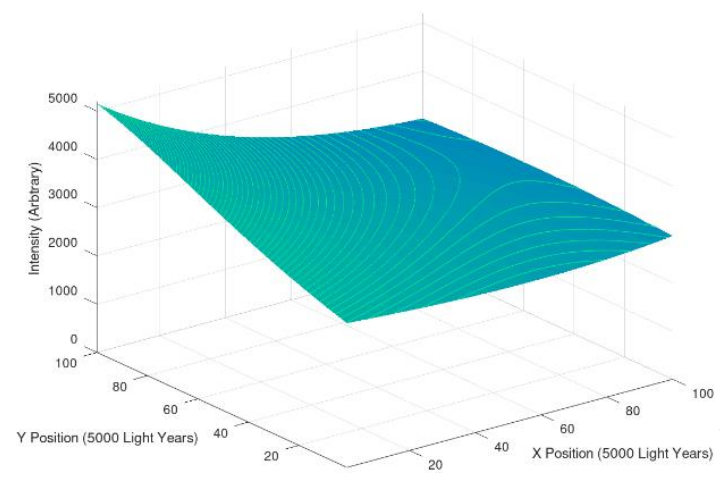

(f)

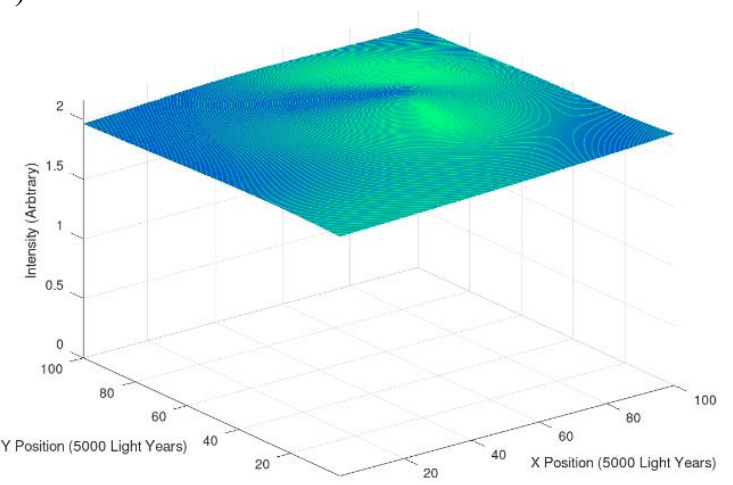

(c)

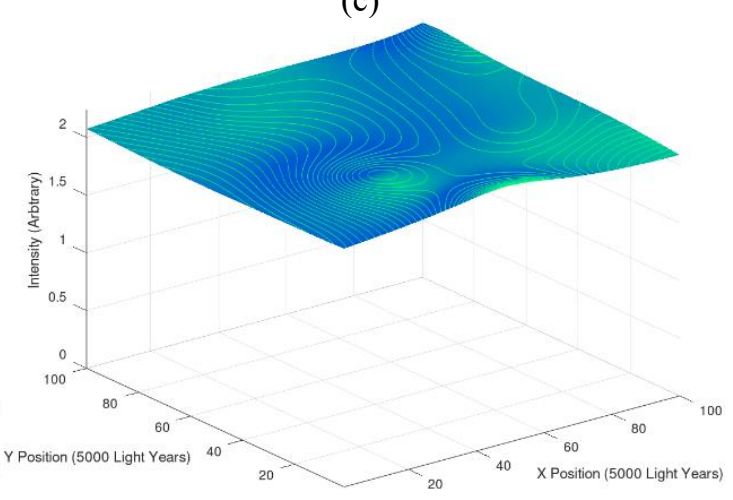

(e)

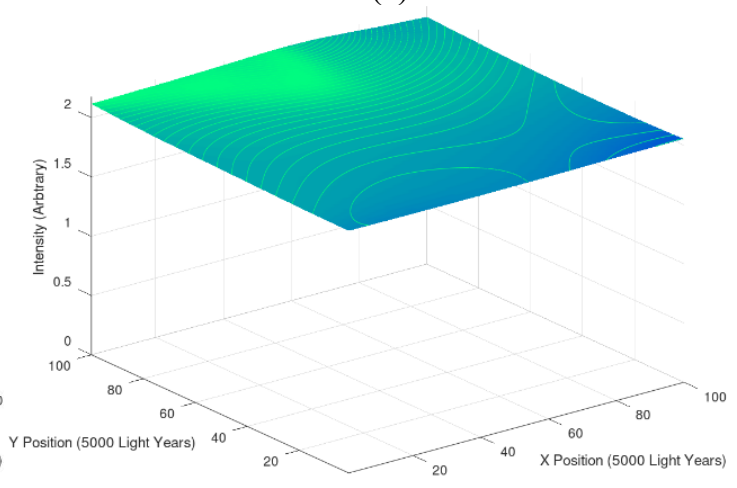

(g) 


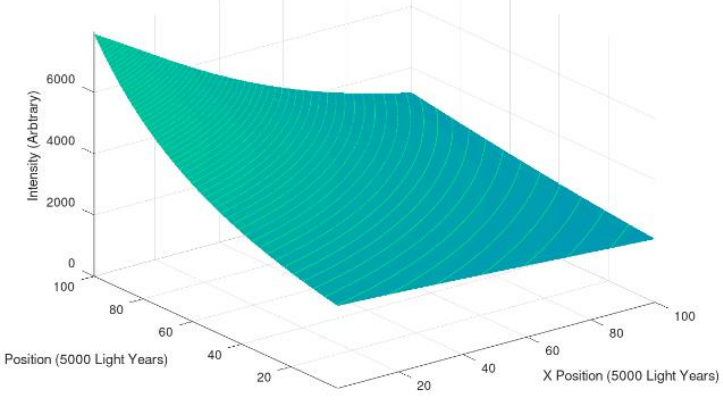

(h)

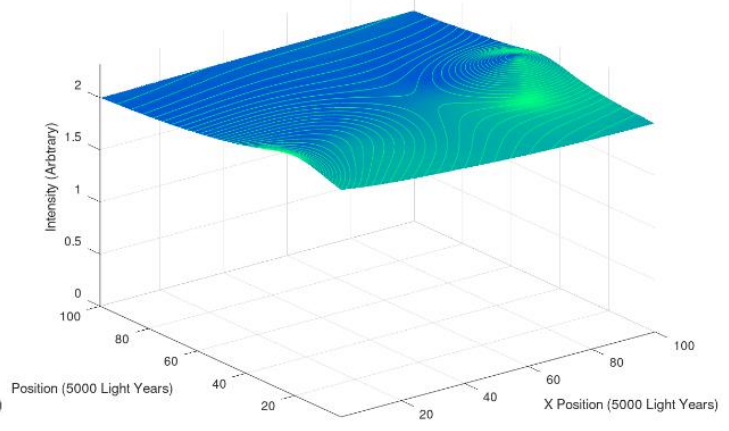

(i)

Figure 3. (a) One demonstration of $10^{4}$ galaxies distributing in a cubic space whose side is 5 million light years. (b) The original intensity distribution including all galaxy contributions. The unit in the $x$ - and $y$ axes are both 5000 light years. (c) The rest intensity distribution after eliminating 10 strongest light sources. The difference between the minimum and the maximum is about $10.0 \%$ in a square region of which the side is 50 thousand light years. (d) The ratio of the maximum to minimum is about 1.4. (e) The difference between the maximum and minimum ia about $8 \%$. (f) The ratio of the maximum to minimum is about 1.7. $(\mathrm{g})$ The difference between the maximum and minimum is about $6 \%$. (h) The ratio of the maximum to minimum is about 3.0. (i) The difference between the maximum and minimum is about $14 \%$.

As a real example, from the picture of the Tokyo Christmas Festival, the blue-white background in the plane close to the ground as shown in Fig. 4. The position of each lamp can be clearly distinguished from each other in the region near the camera, but it is gradually blurred as the distance increases. It involves in the imaging resolution. Such an image is similar to the imaging ability of the Hubble telescope to observe the depths of the universe. If we consider the microwaves radiated from all those $N$ lamps in the picture, it is necessary to exactly know the distribution functions of all microwave sources to establish the radiated microwave intensity distributions in space. When only one light source is turned on and all the others are turned off, a weak microwave intensity distribution is still residual in space. Therefore, as long as one microwave source is un-calculated in the contribution of the radiated microwave intensity distribution in space, it will leave a microwave background intensity distribution. In other words, when we measure the radiated microwave intensity at each point in space, there will be still residual microwave intensity distributed in space by removing the microwave contributions of the $N$-1 lamps. In fact, this residual microwave distribution comes from the un-calculated microwave sources, but it is treated as the so-called microwave background radiation, just similar to the conclusion in Fig. 3(c). Another example is that even if we calculate the contributions of all $N$ lamps, the microwave intensity distribution by subtracting all the $N$ lamps from the measured data still leaves $1 \%$ radiated microwave intensity distribution when the radiation intensity of each lamp is estimated only by $99 \%$ of individual intensity. The residual microwave radiation intensity is distributed in space, and we treat it as the microwave background radiation, which is also similar to the conclusion of Fig. 3(c). 
On the other hand, when we use infrared technology for image reconstruction, it is much hard to distinguish the details of the body, especially when the two bodies are close to each other or overlap, it is usually not easy to distinguish the true appearance of each one. As mentioned previously, the wavelength of the microwave is in the range of $\mathrm{cm}$, and the edge of the microwave image is very blurred usually, so it is impossible to distinguish the galaxies billions of light years far away as clear as the Hubble telescope does. The use of microwaves for omnidirectional detection of the outer space is equivalent to reconstruct an image of $\mu m$-sized bodies by using a source with a wavelength of $\mathrm{cm}$, and the value in each imaging point is a very small part of a very large range with tiny variation. Using the best data and the optimal fitting to obtain the cosmic background radiation by eliminating the main foreground contamination cannot avoid the aforementioned the microwave residue problem. Because any illuminant, especially a cluster of galaxies, has great contribution in the microwave range, from $\mathrm{K}$ band to W-band. If the microwave image is really from light sources far away, the measured spectrum should be the contributions of the multi-illuminants in this band. After eliminating the main contributions of these foreground values from the microwave image, the residue is all the un-calculated galaxies contributing at each measurement point, and thus the almost homogeneous intensity in each direction is obtained similar to the conclusion of Fig. 3(c). It has been considered as the cosmic microwave background radiation after the Big Bang for a very long time. Our research strongly suspects this argument because the total number of galaxies in the universe is unknown and the spatial and time-dependent distribution function of the microwave sources is not accurately established. As long as this distribution function of the microwave sources cannot be accurately established, the error will exist and therefore the residual microwaves will appear which cannot be uniquely determined to be the residual radiation from the Big Bang. More possibly, it is the residual radiation from the un-calculated galaxies and inaccurate estimation of the microwave source strength.

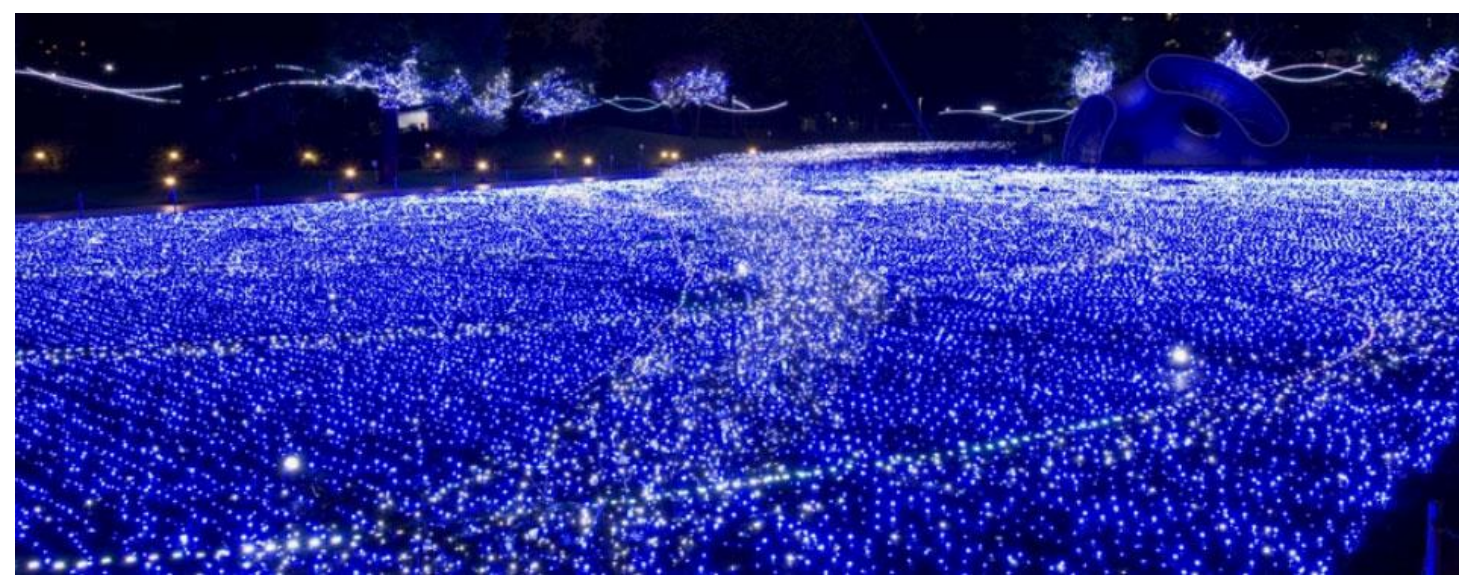

Fig. 4 The light-show in 2009 Tokyo Christmas Festival in which all the lights construct an approximately uniform single-color background [36]. 
The images of the Planck satellites show significantly the resolution better than the COBE satellites, but using microwave to measure the outer space from the place near the Earth has very limited ability to resolve the star clusters or galaxies, so it is not reasonable to conclude that the universe is flat. In the following, we can see what is the problem usin4g the microwave detector. Traditionally, the microwave detector is the horn antenna and the receiving port is an opening with a finite area [30-34] as shown in Fig. 5. In fact, each measured point receives light from different directions, so each pixel on the microwave image represents the summation of all intensities from different directions, and it cannot directly match the intensity distribution of the light source. For example, comparing Fig. 1(a) with Fig. 1(b), the intensity at $23 \mathrm{GHz}$ is explicitly broader than it at $94 \mathrm{GHz}$. When we use the Fig. 1(a) as the intensity distribution of the light source, it probably gives similar microwave imaging at $94 \mathrm{GHz}$. However, it is not true that the microwave image has a similarly wide distribution at $94 \mathrm{GHz}$ in Fig. 1(b). Due to the resolution on the microwave imaging, there are some significant problems that need to overcome [21-25] and using the microwave imaging to construct the cosmic microwave background radiation having the substantial reliability.

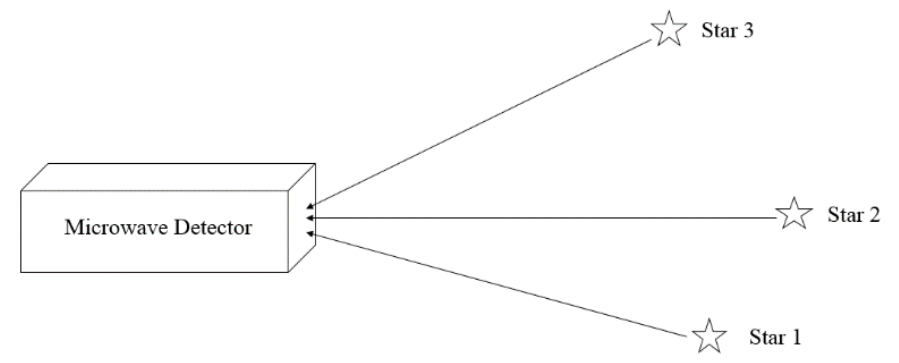

Figure 5. The microwave signals are received by the microwave detector whose opening port is a finite area [30-34], so each measured point receives microwave from different stars at different directions. Each pixel on the microwave imaging represents a lot of contributions from different directions and it cannot directly match the intensity distribution of the microwave sources in the universe.

To subtract the foreground effects, we have to know the galactic and extragalactic spectra. The Cosmic Infrared Background (CIB) spectrum from the COBE/FIRAS observations is represented by this model [7]

$$
I_{C I B}(v)=1.734 \times 10^{-4} T_{C I B}^{3}\left(\frac{v}{v_{0}}\right)^{k_{F}} \frac{\left(\frac{h v}{k T_{C I B}}\right)^{3}}{\exp \left(\frac{h v}{k T_{C I B}}\right)-1}(\mathrm{MJy} / \mathrm{Sr}),
$$

where $T_{C I B}=18.5 \pm 1.2 \mathrm{~K}, v_{0}=3 \times 10^{12} \mathrm{~Hz}$, and $k_{F}=0.64 \pm 0.12$. The other expression, which obtains parameters by using a modified blackbody to fit the Planck data, is [6]

$$
I_{C I B}(v)=0.346\left(\frac{h v}{k T_{C I B}}\right)^{0.86} \frac{\left(\frac{h v}{k T_{C I B}}\right)^{3}}{\exp \left(\frac{h v}{k T_{C I B}}\right)-1}(\mathrm{MJY} / \mathrm{Sr})
$$


where $T_{C I B}=18.8 \mathrm{~K}$ is used here. When we check the above two expressions, something different exists between these two expressions as shown in the following Fig. 6(a). We plot five spectra according to Eqs. (2) and (3), where four spectra are using the parameters $\left(T_{C I B}, k_{F}\right)=(18.5 \pm 1.2 \mathrm{~K}, 0.64 \pm 0.12)$ in Eq. (2) and one spectrum is directly using $T_{C I B}=18.8 \mathrm{~K}$ in Eq. (3). The frequency range is shown from $1 \mathrm{GHz}$ to $10^{4} \mathrm{GHz}$ and five spectra have explicitly different distributions. These two data collected in different time and separated by about 20 years. It means that the CIB foreground spectrum is inconsistent in different time. Even at the same time like it in Eq. (2), the uncertainties in $T_{C I B}$ and $k_{F}$ mean that the spectrum cannot be uniquely and precisely determined what we specially emphasize here. The inaccurate estimation exists so as to lead the microwave background radiation appears. Let's speak more clearly. When we consider the difference between these two equations at the same $T_{C I B}$, it is

$$
\Delta I_{C I B}(v)=\left.I_{C I B}(v)\right|_{T_{C I B}=18.8 \mathrm{~K}}-\left.I_{C I B}(v)\right|_{\substack{T_{C I B}=18.8 \mathrm{~K} \\ k_{F}=0.76}}
$$

Although the $T_{C I B}$ in Eq. (3) is within the range in Eq. (2), it is very explicitly that the two powers of the frequency $v$ in the bracket are different. One is 0.76 and the other is 0.86. The difference between the two spectra in Eq. (4) is plotted in Fig. 6(b). We can suppose these two spectra that one is the real radiation spectrum and the other is inaccurately estimated spectrum. This difference is analog to our statement that inaccurate estimation of the microwave source strength causes the residual microwave background radiation.

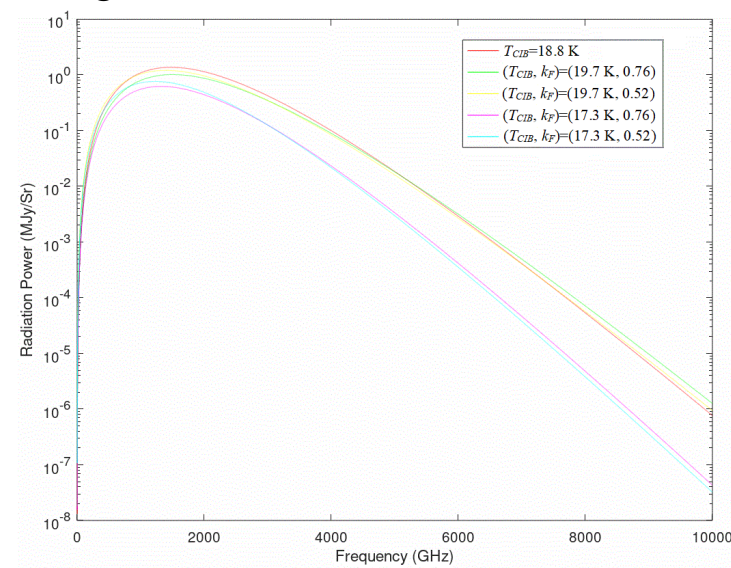

(a)

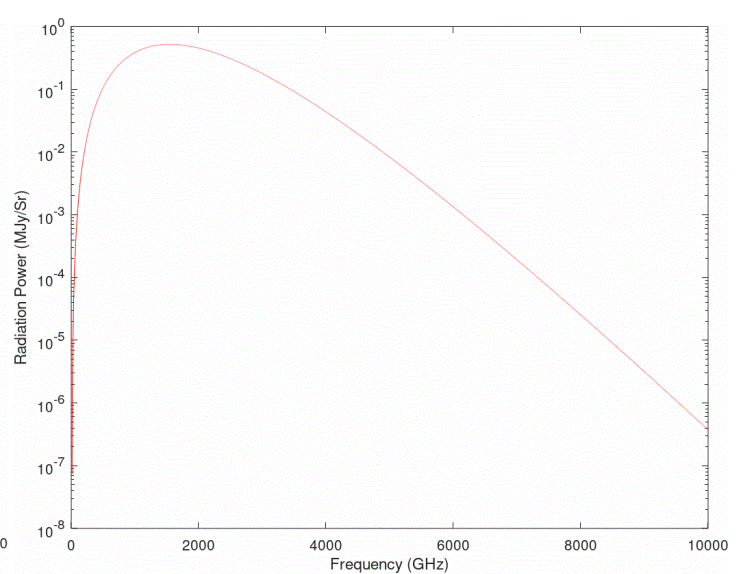

(b)

Figure 6. (a) Four spectra using the parameters $\left(T_{C I B}, k_{F}\right)=(18.5 \pm 1.2 \mathrm{~K}, 0.64 \pm 0.12)$ in Eq. (2) and one spectrum using $T_{C I B}=18.8 \mathrm{~K}$ in Eq. (3) are plotted. (b) The difference between two spectra is plotted. Both $T_{C I B}$ are the same at $18.8 \mathrm{~K}$. The two powers of the frequency $v$ in the bracket are different where one is 0.76 and the other is 0.86 .

The CIB foreground spectrum extracted from the FIRAS sky spectra was an obvious example where the FIRAS spectra are functions of frequency and the galactic coordinate [7]. It can also be seen from our simulations that the 10,000 galaxies build small anisotropic intensity distributions in the detection plane. It is always not flat so 
the CIB and CMB spectra must be functions of the observation coordinates. Therefore, we propose an spatial function for the CMB spectrum in the observation coordinates $(x, y)$ expanding in the Fourier series

$$
\begin{aligned}
f(x, y)=\frac{A B_{00}}{4} & \\
& +\sum_{n=1}^{\infty} \sum_{m=1}^{\infty}\left[A B_{n m 1} \sin \left(\frac{n \pi x}{L}\right) \sin \left(\frac{m \pi y}{L}\right)\right. \\
& +A B_{n m 2} \sin \left(\frac{n \pi x}{L}\right) \cos \left(\frac{m \pi y}{L}\right)+A B_{n m 3} \cos \left(\frac{n \pi x}{L}\right) \sin \left(\frac{m \pi y}{L}\right) \\
& \left.+A B_{n m 4} \cos \left(\frac{n \pi x}{L}\right) \cos \left(\frac{m \pi y}{L}\right)\right],
\end{aligned}
$$

where $A B_{00}, A B_{n m 1}, A B_{n m 2}, A B_{n m 3}$, and $A B_{n m 4}$ are coefficients, $n$ and $m$ are both positive integers, and $L=25,000$ light years. Then we use Fig. $3(\mathrm{~g})$ as an example to find out the approximate form when $n$ and $m$ are limited to 10 . Substituting the intensity distribution of Fig. 3(g) into the above Fourier expansion series, it gives the approximation by considering the 15 largest coefficient terms. Therefore, the approximate function of the intensity distribution at a certain frequency in the two-dimensional $(x, y)$ plane can be expressed as follows

$$
\begin{gathered}
f(x, y) \approx 2.057-0.003131 \cos \left(\frac{\pi x}{L}\right) \sin \left(\frac{\pi y}{L}\right)-0.0010101 \sin \left(\frac{\pi x}{L}\right) \sin \left(\frac{3 \pi y}{L}\right) \\
+\left[0.0022277 \sin \left(\frac{\pi x}{L}\right)-0.0016121 \sin \left(\frac{2 \pi x}{L}\right)+0.0011489 \sin \left(\frac{3 \pi x}{L}\right)\right] \sin \left(\frac{2 \pi y}{L}\right) \\
+\left[0.0057951 \sin \left(\frac{\pi x}{L}\right)-0.0041816 \cos \left(\frac{\pi x}{L}\right)\right. \\
-0.004268 \sin \left(\frac{2 \pi x}{L}\right)+0.0029141 \sin \left(\frac{3 \pi x}{L}\right) \\
-0.0021795 \sin \left(\frac{4 \pi x}{L}\right)+0.0017446 \sin \left(\frac{5 \pi x}{L}\right) \\
-0.001457 \sin \left(\frac{6 \pi x}{L}\right)+0.0012525 \sin \left(\frac{7 \pi x}{L}\right) \\
\left.-0.0010994 \sin \left(\frac{8 \pi x}{L}\right)\right] \cos \left(\frac{\pi y}{L}\right) .
\end{gathered}
$$

In this expression, the zero-order term is 2.057 , which represents the average value of the entire two-dimensional detection plane after removing 10 extremely strong light sources, similar to the one possible distribution of the homogeneous CMB. The sum of the higher-order terms after subtracting the zero-order term represents the local fluctuations, which is similar to the anisotropy in the CMB. That is to say, the foreground distribution at a certain frequency is not merely represented by a smooth function such as Eq. (2) or (3). It can be improved by a spatial correction function to better represent the residual effect of all the microwave radiation sources. The $3 \mathrm{D}$ image and contour lines drawn by the function in Eq. (6) are shown in Fig. 7. It can be seen 
that there are peaks and valleys, where the largest peak is about 0.0189 unit, and the lowest valley is -0.0178 unit. The intensity distribution described in Eq. (6) has fluctuations of about $\pm 0.9 \%$ relative to the average value.

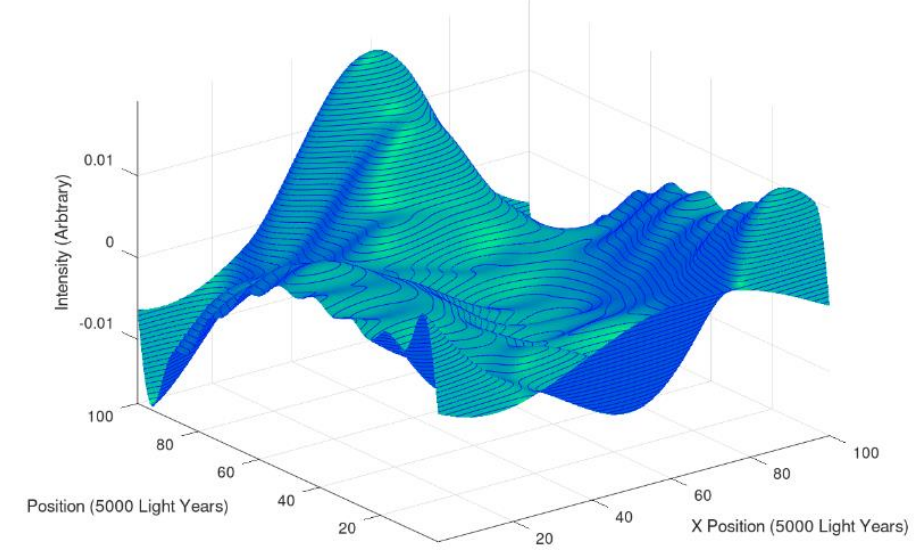

Figure 7. The rest intensity distribution in Fig. 3(g) redrawn by the high-order Fourier series terms. It is analog to the anisotropy in the Cosmic Microwave Background distribution.

Similarly, using the Fourier expansion series of Eq. (5), we can get the approximate representation of Fig. 3(i). Considering the 26 largest coefficient terms, the approximate function of the intensity distribution at a certain frequency in the two-dimensional $(x, y)$ plane can be expressed as follows

$$
\begin{aligned}
& f(x, y) \approx 2.0827 \\
& +\left[0.03262 \sin \left(\frac{\pi x}{L}\right)+0.015222 \cos \left(\frac{\pi x}{L}\right)\right. \\
& -0.012485 \sin \left(\frac{2 \pi x}{L}\right)+0.010060 \cos \left(\frac{2 \pi x}{L}\right) \\
& \left.-0.0057294 \sin \left(\frac{4 \pi x}{L}\right)\right] \sin \left(\frac{\pi y}{L}\right) \\
& +\left[0.016575 \sin \left(\frac{\pi x}{L}\right)-0.013089 \cos \left(\frac{\pi x}{L}\right)\right] \cos \left(\frac{\pi y}{L}\right) \\
& +\left[0.0094993 \sin \left(\frac{2 \pi x}{L}\right)-0.0087329 \cos \left(\frac{2 \pi x}{L}\right)\right] \sin \left(\frac{2 \pi y}{L}\right) \\
& +\left[-0.0087329 \sin \left(\frac{2 \pi x}{L}\right)+0.0055612 \cos \left(\frac{2 \pi y}{L}\right)\right] \sin \left(\frac{3 \pi y}{L}\right) \\
& +\left[-0.014410 \cos \left(\frac{2 \pi y}{L}\right)+0.011505 \cos \left(\frac{3 \pi y}{L}\right)-0.0098784 \cos \left(\frac{4 \pi y}{L}\right)\right. \\
& +0.0083912 \cos \left(\frac{5 \pi y}{L}\right)-0.0071705 \cos \left(\frac{6 \pi y}{L}\right) \\
& \left.+0.0062107 \cos \left(\frac{7 \pi y}{L}\right)-0.0054651 \cos \left(\frac{8 \pi y}{L}\right)\right] \sin \left(\frac{\pi x}{L}\right)
\end{aligned}
$$




$$
\begin{aligned}
+[-0.017599 & \sin \left(\frac{2 \pi y}{L}\right)+0.010237 \sin \left(\frac{3 \pi y}{L}\right)-0.0057739 \sin \left(\frac{4 \pi y}{L}\right) \\
+ & 0.0017446 \sin \left(\frac{5 \pi x}{L}\right)-0.0014570 \sin \left(\frac{6 \pi x}{L}\right) \\
+ & 0.0012525 \sin \left(\frac{7 \pi x}{L}\right) \\
& \left.-0.0010994 \sin \left(\frac{8 \pi x}{L}\right)\right] \cos \left(\frac{\pi x}{L}\right) .
\end{aligned}
$$

In this expression, the value of the zero-order term is 2.0827 , which is $1.25 \%$ slightly higher than the average value in Fig. 7, which is also similar to the one possible distribution of homogeneous CMB. The sum of higher-order terms after subtracting the zero-order term also similar to the anisotropy in CMB. The 3D image and contour lines drawn by the function in Eq. (7) are shown in Fig. 8. It can be seen that there are peaks and valleys, the largest peak is about 0.0969 unit, and the lowest valley is -0.0817 units. The fluctuations in this example are obviously larger than those in Fig. 7. The intensity distribution described in Eq. (7) has fluctuations of $+4.6 \%$ and $-4.0 \%$ relative to the average. Therefore, the average values obtained from different $10^{4}$ galaxy distributions are very similar, but they show fluctuations in their spatially microwave radiation distributions.

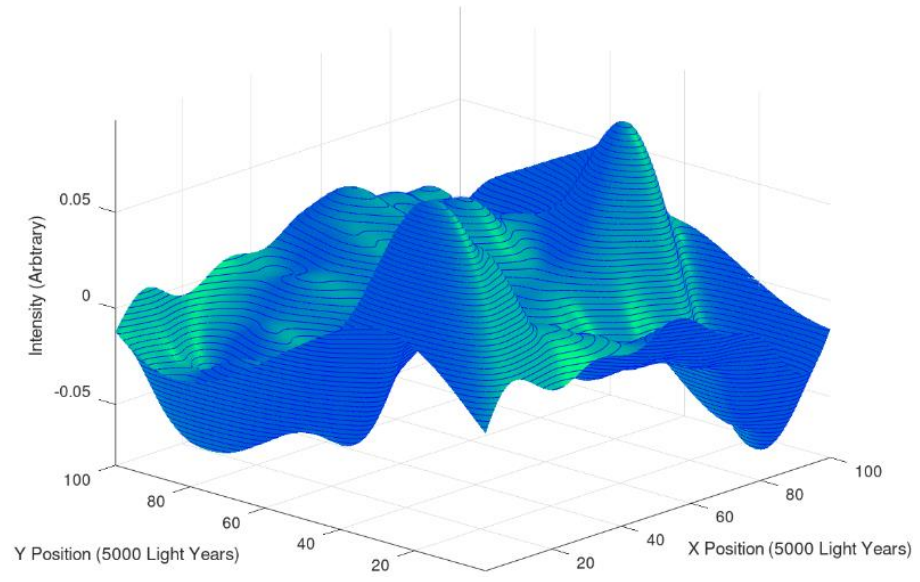

Figure 8. The rest intensity distribution in Fig. 3(i) redrawn by the high-order Fourier series terms. It is analog to the anisotropy in the Cosmic Microwave Background distribution.

The phenomenon described in Fig. 4 can also be mathematically expressed in Eq. (5) similar to Eqs. (6) and (7). When we plot the intensity of all the light sources in Fig. 4 on a certain detection plane, it is possible to obtain similar graphs as shown in Figs. 3(c), (e), (g), and (i). All the light sources in Fig. 4 can be assumed to have the same luminance, and there are no particularly strong light sources there. This description is equivalent to the situation after the main strongest light sources are removed to obtain the CMB what we discuss here. To do the Fourier series expansion on the intensity of the detection plane, then we will get a mathematical expression like Eq. (6) or (7). 
Regardless of the really subtle luminant differences in each light source, the overall effect is equivalent to the zero-order term $A B_{00}$ in Eq. (5). After deducting the contributions of all these light sources with the same luminance, high-order terms will be left to form a similar distribution as in Figs. 7 and 8. The existence of these higherorder terms is due to the luminance of the light source slightly different from each other. Therefore, when applying this concept to the observation of cosmic microwave radiations, as long as the source cannot be accurately known, similar phenomena will occur. Especially, after removing the main foreground pollutions, the result is a uniform CMB with tiny anisotropy. As mentioned before, all our results from Eqs. (5) to (8) are scalable where $L=25,000$ light years is a demonstration. In fact, $L$ is a parameter assigned by the discussing case. If we reduce $L$ from 25,000 light years to $10,000 \mathrm{~km}$, Figs. 7 and 8 can be a case to represent the tiny CMB anisotropy in our observation coordinates. When we detect the microwave radiation from whole the sky on the Earth, each detecting pixel represents the contributions of many galaxies and is the total performance like it in Figs. 7 and 8. If we observe the microwave radiation at a fixed place on the Earth, it is just the case to choose one observation place $(x, y)$ in Figs. 7 and 8. Then, if there are $10^{8}$ pixels in the distribution, we shall have $10^{8}$ data at the same place extracting from the $10^{8}$ microwave fluctuation distributions like them in Figs. 7 and 8. Finally, we will get the omnidirectional CMB anisotropic distribution.

Except for the above discussions, the third possibility is that we directly detect the blackbody radiation of the dilute ultracold gas in space surrounding the Earth.

\section{Conclusions}

Considering the basic microwave imaging characteristics, we explain the representation of the cosmic microwave radiation image. Due to the limitation of resolution, it cannot clearly exhibit the distribution of 200 million galaxies in the universe, especially the lower frequency measurements are more blurred. Due to the finite-area opening on the microwave measuring instrument, the cosmic microwave measurement is the superposition of the incident microwaves from different directions, and the image does not correspond to the actual cosmic microwave source. The analysis of the cosmic microwave image is the problem of reliability. From a simulation including 10,000-point sources with random distribution and random intensity in a finite space, we know that a uniform radiation intensity distribution over a large range by the remaining point sources after removing the several strongest point sources. Therefore, Cosmic Microwave Background radiation cannot be uniquely interpreted as a uniform microwave radiation full of the entire space which was left after the initial formation of the universe. More possibly, it is the residual radiation from the uncalculated galaxies and inaccurate estimation of the microwave source strength. 
When we say that the Cosmic Microwave Background radiation is extremely high isotropy, it means that the Cosmic Microwave Background radiation does not come from a local source. Regardless of gravity, light travels along the geodesic. Mostly, light propagates along a straight line in an empty space. When we observe galaxies in the sky, the farther the distance is, the closer the effect is to the point light source. The results of astronomical observations can point out the radiation sources, just as we observe some stars producing gamma-ray bursts. Therefore, this omnidirectional and uniform Cosmic Microwave Background radiation raises questions about the Big Bang. In the 1987A supernova event, it also tells us that it is almost impossible to measure the microwave radiation of 13.8 billion years after the Big Bang. Electromagnetic waves transmitted from the far distant galaxies travel at the speed of light, and as soon as they are generated, they travel forward to the edge of the universe at the speed of light. The microwaves 13.8 billion years ago have already traveled a distance of 13.8 billion lightyears, if they are not absorbed by other substances. Thus, how can the very empty universe keep the microwave radiation that moved at the speed of light 13.8 billion years ago?

As the results of the aforementioned B-mode polarization signal show, incorrect estimation of the radiation source leads to incorrect interpretation. When we discover more potential factors, the early observation conclusions 50 years ago may be dramatically different. The possible explanation for such a precise blackbody radiation spectrum with almost negligible error is to directly measure some things of such temperature. In particular, the result of the isotropy of the whole space indicates that the sources with this temperature is homogeneously distributed in the universe. Therefore, another explanation is to directly measure the blackbody radiation of the dilute uniform ultracold atomic and molecular gases surrounding the Earth in space!

\section{Acknowledgement}

This research is under no funding.

\section{References:}

1. A. A. Penzias and R. W. Wilson, "A Measurement of Excess Antenna Temperature at $4080 \mathrm{Mc} / \mathrm{s}$," Astrophys. J. 142, 419 (1965).

2. D. J. Fixsen, E. S. Cheng, J. M. Gales, J. C. Mather, R. A. Shafer, and E. L. Wright, "The Cosmic Microwave Background Spectrum From The Full COBE ${ }^{1}$ FIRAS Data Set," Astrophys. J. 473, 576 (1996).

3. D. J. Fixsen, "The Temperature of The Cosmic Microwave Background," Astrophys. J. 707, 916 (2009).

4. https://en.wikipedia.org/wiki/Cosmic microwave background\#: : text $=$ The $\% 20$ cosmic $\% 20$ micro wave \%20background\%20(CMB,background\%20radiation\%20filling\%20all\%20space.

5. G. Hinshaw et al., "Five-Year Wilkinson Microwave Anisotropy Probe (WMAP1) Observations: Data Processing, Sky Maps, And Basic Results,” Astrophys. J. Suppl. Ser. 180, 225 (2009).

6. Maximilian H. Abitbol, Jens Chluba, J. Colin Hill, and Brandley R. Janson, "Prospects for Measuring Cosmic Microwave Background Spectral in the Presence of Foregrounds," MNRAS 471, 
1126-1140 (2017).

7. D. J. Fixsen, E. Dwek, J. C. Mather, C. L. Bennett, and R. A. Shafer, "The Spectrum of the extragalactic far infrared background from the COBE FIRAS observations," Astrophys. J. 508, 123 (1998).

8. G. De Zotti, M. Negrello, G. Castex, A. Lapi, and M. Bonato, "Another Look at Distortions of Cosmic Microwave Background Spectrum," Journal of Cosmology and Astroparticle Physics 03, 047 (2016).

9. Vadim R. Munirov and Nathaneil J. Fisch, "Radiation in Equilibrium with Plasma and Plasma Effect on Cosmic Microwave Background," Physical Review E 100, 023202 (2019).

10. Daneil Aloni, Kfir Blum, and Rapheal Flauger, "Cosmic Microwave Background Constraints on Primordial Black Hole Dark Matter," Journal of Cosmology and Astroparticle Physics 05, 017 (2017).

11. Suzanne Staggs, Joe Dunkley, and Lyman Page, "Recent Discoveries from the Cosmic Microwave Background: A Review of Recent Progress,” Rep. Prog. Phys. 81, 044901 (2018).

12. R. A. Sunyaev and R. Khatri, Unavoidable CMB spectral features and blackbody photosphere of our universe Thirteenth Marcel Grossmann Meeting: On Recent Developments in Theoretical and Experimental General Relativity, Astrophysics and Relativistic Field Theories ed K Rosquist, R T Jantzen and R Ruffini (Singapore: World Scientific) pp 373-97 (2015).

13. J. C. Hill, N. Battaglia, J. Chluba, S. Ferraro, E. Schaan, and D. N. Spergel, Phys. Rev. Lett. 115, 261301 (2015).

14. Nia Imara and Abraham Loeb, "The Distortion of the Cosmic Microwave Background Spectrum Due to Intergalactic Dust,” Astrophys. J. 825, 130 (2016).

15. Ade, P.A.R. et al (BICEP2 Collaboration), "Detection of B-Mode Polarization at Degree Angular Scales by BICEP2,” Phys. Rev. Lett. 112, 241101 (2014). doi:10.1103/PhysRevLett.112.241101.

16. A. Lewis and A. Challinor, "Weak gravitational lensing of the CMB," Physics Reports 429, 1-65 (2006). doi:10.1016/j.physrep.2006.03.002.

17. Planck Collaboration, "Planck Intermediate Results XXX. The Angular Power Spectrum of Polarized Dust Emission at Intermediate and High Galactic latitudes," A\&A 586, A133 (2016).

18. P. A. R. Ade et al., "Joint Analysis of BICEP $2 /$ Keck Array and Planck Data," Physical Review Letters 114, 101301 (2015).

19. Nils W. Halverson, “A Clearer View of a Duty Sky,” Physics 8, 21 (2015).

20. E. J. Bond, X. Li, S. C. Hagness, and B. D. Van Veen, "Microwave Imaging Vis Space-Time Beamforming For Early Detecting Breast Cancer," IEEE Trans. Antennas Propagat. 51, 1690 (2003).

21. S. Kharkovsky and R. Zoughi, "Microwave And Millimeter wave Nondestructive Testing And Evaluation - Overview And Recent Advances,” IEEE Instrum. Meas. Mag. 10, 26 (2007).

22. Karsten J. Siebert, Holger Quast, Rainer Leonhardt, Torsten Lo“ ffler, Mark Thomson, Tobias Bauer, Hartmut G. Roskos, and Stephanie Czasch, "Continuous-Wave All-Optoelectronic Terahertz Imaging," Appl. Phys. Lett. 80, 3003 (2002).

23. Nicholas Karpowicz, Hua Zhong, Jingzhou Xu, Kuang-I Lin, Jenn-Shyong Hwang, and X-C Zhang, "Comparison Between Pulsed Teraherz Time-Domain Imaging And Continuous Wave Terahertz Imaging," Semicond. Sci. Technol. 20, S293 (2005).

24. Torsten Lo"ffler, Karsten J. Siebert, Noboru Hasegawa, Tobias Hahn, and Hartmut G. Roskos, "AllOptoelectronic Terahertz Imaging Systems And Examples of Their Application,” Proc. IEEE 95, 1576 (2007).

25. Torsten Löffler, Thilo May, Christian am Weg, Ali Alcin, Bernd Hils, and Hartmut G. Roskos, “Continuous-Wave Terahertz Imaging With A Hydrid System,” Appl. Phys. Lett. 90, 091111 (2007).

26. Sloan Digital Sky Survey. The Scope of the Ninth SDSS Data Release (DR9). http://www.sdss3.org/dr9/scope.php. Accessed 2 April 2013.

27. Shadab Alam et al., "The Eleventhh And Twelfth Data Releases of Sloan Digital Sky Surveys: Final Data From SDSS-III,” Astrophys. J. 219, 12 (2015).

28. R. P. Saglia et al. "The Photometric Classification Serve For Pan-STARRS1," Astrophys. J. 746, 128 (2012).

29. https://inhabitat.com/largest-ever-new-map-of-universe-shows-1-2-million-galaxies/.

30. Stafford Withington and J. Anthony Murphy, "Analysis of Diagonal Horn Through Gauss-Hermite Modes," IEEE Trans. Antennas Propagat. 40, 198 (1992).

31. Joakim F. Johansson and Nicholas D. Whyborn, "The Diagonal Horn As A Sub-Millimeter Wave Antenna,” IEEE Trans. Microw. Theory Tech. 40, 795 (1992).

32. V. N. Tiwari, T. Tiwari, S. P. Singh, and R. K. Jha, "Near Field Distribution of Solid Dielectric Diagonal Horn Antenna," Int. J. Electronics 82, 167 (1997).

33. V. N. Tiwari, T. Tiwari, S. p. Singh, and R. K. Jha, "Far Field Patterns of Solid Dielectric Diagonal 
Horn Antenna,” Int. J. Electronics 82, 175 (1997).

34. T. Tiwari and V. N. Tiwari, "Near Field Distributions And Radiation Patterns of Hollow Dielectric Wall Pyramidal Horn Antennas," Int. J. Electronics 84, 383 (1998).

35. K. Hirata et al., "Observation of A Neutrino Burst From The Supernova SN 1987A," Phys. Rev. Lett. 58, 1490 (1987).

36. https://tokyo5.files.wordpress.com/2009/12/midtown-blue.jpg. 